\title{
Simulation of the Temperature Dependence of the Density of States in a Strong Magnetic Field
}

\author{
G. Gulyamov1, U. I. Erkaboev¹, N. Yu. Sharibaev,2 \\ ${ }^{1}$ Namangan Engineering Pedagogical Institute, Namangan, Uzbekistan \\ ${ }^{2}$ Namangan Engineering Institute of Technology, Namangan, Uzbekistan \\ Email: Gulyamov1949@mail.ru
}

Received 31 December 2013; revised 28 January 2014; accepted 25 February 2014

Copyright (C) 2014 by authors and Scientific Research Publishing Inc.

This work is licensed under the Creative Commons Attribution International License (CC BY). http://creativecommons.org/licenses/by/4.0/

c) (i) Open Access

\section{Abstract}

The temperature dependence of the density of states in strong magnetic fields. On the basis of the model constructed, a computer program calculating the density of electronic states in a quantizing magnetic field. Used new, based on quantum statistics, the approach to the calculation of the temperature dependence of the density of states in a strong magnetic field. Mathematical modeling of the density of states using the experimental values of a continuous density of states makes it possible to calculate the Landau levels.

\section{Keywords}

Cyclotron Frequency and Effective Mass, A Quantizing Magnetic Field, Landau Levels, The Energy Spectrum, The Numerical Simulation and Experiment

\section{Introduction}

In a strong magnetic field, the energy spectrum of electrons in a semiconductor or metal becomes quantized, so that the density of energy states as a function of energy acquires an oscillating character. It is this fact is the origin of the oscillatory magnetic field dependence of a number of equilibrium and no equilibrium quantities characterizing the state and behavior of the electrons in the crystal in a quantizing magnetic field [1]. In [2] [3] investigated the dependence of the longitudinal resistance and static magnetic susceptibility of the magnetic field perpendicular to the plane over a narrow silicon quantum well bounded by $\delta$-barriers heavily doped with boron detected oscillations of the Shubnikov-de Haas and de Haas-van Alphen at high temperatures in weak magnetic fields. 
[4]-[7] calculated the temperature dependence of the density of states defined by relaxation spectroscopy of the energy levels in semiconductors. It is shown that due to the thermal broadening of the levels of a discrete spectrum with hanging temperature turns into a continuous energy spectrum. With the expansion of the energy spectrum density of states as functions $G N$ (GN-energy derivative of the probability of the devastation of the discrete level), it was shown that the magnitude of the energy gap is temperature dependent. These works have not considered the effect of temperature on the density of energy states in a strong magnetic field.

The aim of this work is to develop a new approach to the explanation of the temperature dependence of the density of states in a strong magnetic field.

\section{Model}

The temperature dependence of the density of states due to thermal broadening of discrete energy states [4]-[7]. Thermal broadening can be described by the temperature dependence of the occupation probabilities of the energy levels. Time thermal emission of electrons from deep levels $E_{i}$ in the allowed band energy $E$ is determined by the exponential factor $\exp \left(\frac{E-E_{i}}{k T}\right)$ and the probability of deep desolation filled levels depends exponentially on the energy of the state and the sample temperature.

To describe the temperature dependence of the density of states, we assume that the density of states at zero temperature known function of energy $N_{s}\left(E_{0}\right)$. In particular, for a free electron gas in a magnetic field density of states is determined by the following expression [8]

$$
N_{H}(E, H)=\frac{1}{4 \pi^{2}}\left(\frac{2 m^{*}}{\hbar^{2}}\right)^{3 / 2} \hbar \omega_{H} \sum_{n}\left\{E-\left(n+\frac{1}{2}\right) \hbar \omega_{H}\right\}^{-1 / 2}
$$

Here, $\omega_{H}$-cyclotron frequency, $m^{*}$ and $E$-effective mass and the energy of a free electron, $n$-number of Landau levels.

Each state with energy $E_{i}$ broadens with increasing temperature. Temperature broadening of the state with energy $E_{i}$ describes statistics Shockley-Read-Hall. Meaning broadening described derivative energy on the content of the devastation $\rho$ the state with energy $E$ [5]

$$
\frac{\partial \rho\left(E_{0}, E, T\right)}{\partial E_{0}}=G N\left(E_{0}, E\right)=\frac{1}{k T} \exp \left(\frac{1}{k T}\left(E-E_{0}\right)-\exp \left(\frac{1}{k T}\left(E-E_{0}\right)\right)\right)
$$

$E_{0}$-energy parameter of the time-dependent. The resulting density of states, taking into account the contribution of thermal broadening of all states, will be described by the sum of all of the broadening in the energy region. Mathematically, this reduces to the expansion in the number density of states $N_{s}(E, H, T)$ at a temperature $T$ by $G N$-functions. Moreover, the coefficient of expansion is the energy density of states, which does not account for thermal broadening of the levels. At absolute zero, $G N$-function turns into the Dirac delta function [5]-[7]. When the temperature tends to absolute zero density of states turn to the expansion coefficients in a series of $G N$-functions. If you know the density of states at finite temperature $N_{s}(E, T)$, then using a series expansion in $G N$-functions can calculate the density of states at any temperature. In [5], it was shown that the continuous spectrum defined at room temperature, are formed by the thermal broadening of the number of discrete levels in the band gap. Thermal broadening of the energy states of the conduction band and valence band gap of the semiconductor explains the temperature dependence of the band gap of the semiconductor [6].

\section{The Temperature Dependence of the Density of States in a Strong Magnetic Field}

Dependence of the energy $E$ of the electron with ellipsoidal quadratic dispersion law in a magnetic field of principal quantum number $n$, the number of quanta of spin $\mathrm{s}$ and projection $p_{z}$ momentum on the direction of the magnetic field $H$ takes the following form [9]:

$$
E\left(n, s, p_{z}\right)=\hbar \omega_{c}\left(n+\frac{1}{2}\right)+\frac{1}{2} s g \mu_{B} \mu_{0} H+\frac{p_{z}^{2}}{2 m_{z}^{*}}
$$


Here, $g$-factor is defined only in the orientation of the magnetic field $H$ and does not depend on the projection of the momentum $p_{z}, \mu_{B}$ - Bohr magnetrons, $m_{z}$-longitudinal effective mass.

The total density of energy states in a magnetic field in an electronic system with a quadratic isotropic dispersion law excluding spinal splitting of the Landau levels can be written as [9]:

$$
N_{H}(E, H)=\frac{m^{3 / 2} \hbar \omega_{c}}{\sqrt{2} \pi^{2} \hbar^{3}} \sum_{n=0}^{n_{E}}\left[E-\hbar \omega_{c}\left(n+\frac{1}{2}\right)\right]^{-1 / 2}
$$

where, $E$-energy of a free electron, $n=0,1, \cdots$-number of Landau levels, $m$-cyclotron mass, $\omega_{c}=\frac{e H}{m c}-$ cyclotron frequency.

With a decrease in the density of the magnetic field $H$ increases Landau parabolas. Obviously, in the limit $H \rightarrow 0$ we need from discrete parabolas Landau again go to the quasi-continuous spectrum of three-dimensional system $E=E\left(p_{x}, p_{y}, p_{z}\right)$.

In particular, if the energy spectrum of a purely discrete (point), the density of energy states is the sum of $\delta$ functions concentrated at the points of the spectrum of $E_{i}$, whose amplitude $N_{s i}=\psi_{i}^{2}(0)+\psi_{i}^{\prime 2}(0)$, where $E_{i} u \psi_{i}(x)$-normalized to unity own functions [10]:

$$
N_{s}(E)=\sum_{i} N_{s i} \delta\left(E-E_{i}\right)
$$

Thermal broadening of the levels in the magnetic field leads to a smoothing of discrete levels. Thermal broadening is taken into account using the GN function. As in [4]-[7], $N_{s}(E, H, T)$ in a series in GN functions

$$
N_{s}(E, H, T)=\sum_{i=1}^{n} N_{s i}\left(E_{i}, E, H\right) G N\left(E_{i}, E, T\right)
$$

where, $N_{s i}\left(E_{i}, H, E\right)$ - density of states in a quantizing magnetic field at absolute zero temperature.

Considered energy interval in the allowed bands and the band gap divide into equal smaller parts. Summing the formula (5) we obtain $N_{s}(E, H, T)$ temperature dependent. This expression when $T \rightarrow 0$ transformed into (3). In this case, the Landau levels appear sharply (in Figure 1).

With increasing temperature starts to smooth sharp spikes and oscillations of the density of states are gradually disappearing. At sufficiently high temperatures $k T>\hbar \omega, N_{s}(E, H, T)$ is converted into a solid state density in zero magnetic field. Figure 1 shows the density of states in a magnetic field calculated series expansion in $G N$ functions, functions for the derivative of the Fermi-Dirac distribution function and Gauss, $\hbar \omega_{c}=0.01{ } B$ at temperature $T=0.4 \mathrm{~K}, k T<\hbar \omega_{c}$. At such low temperatures the effect of thermal broadening of the weak and the density of states does not feel Ns deviations from the ideal form, which does not account for the influence of temperature.

Figure 2 shows $N_{s}(E, H)$ the temperature to $10 \mathrm{~K}$. As can be seen from these figures, with increasing temperature, sharp peaks of the Landau levels are gradually smoothed. At a temperature of $35 \mathrm{~K}$ peaks of the Landau levels is almost invisible, and coincides with the density of states of expansion in the Gaussian function and the derivative function of the Fermi-Dirac (in Figure 3).

Change $\omega_{c}=\frac{e H}{m^{*} c}$ alters the energy separation between the peaks of the Landau levels. Thus, the oscillations of the density of states is observed at temperatures $k T \ll \hbar \omega_{c}$. Beginning with temperatures on the order $0.5 k T \sim \hbar \omega_{c}$ of the oscillations of the density of states due to the Landau quantization is observed. In this case, the measurements provide a continuous range of density of states. For the detection of quantum levels necessary to solve the inverse problem. In this case, it is necessary to find a discrete level due to the Landau quantization. For this it is necessary to measure $N_{s}(E, H, T)$ the temperature of the experiment, followed by calculation to obtain $N_{s}(E, H, T)$ at low temperatures.

Methods of determining the $N_{s}$ can be constructed as follows. Experimentally $N_{s}(E, H, T)$ determined for two different temperatures $T_{1}$ and $T_{2}$, so that it was the same for both $N_{s}\left(E, H, T_{1}\right)$ and $N_{s}\left(E, H, T_{2}\right)$. The same measurements and calculations can be done for several values of the magnetic fields $H_{1}$ and $H_{2}$. To improve the accuracy of $N_{s}(E, H)$ you can use the results found experimentally $N_{s}(E, H, T)$ more than three or four different values of temperature. If the simulation results $N_{s}(E, H, T)$ for various values of $T$ and $H$ are 


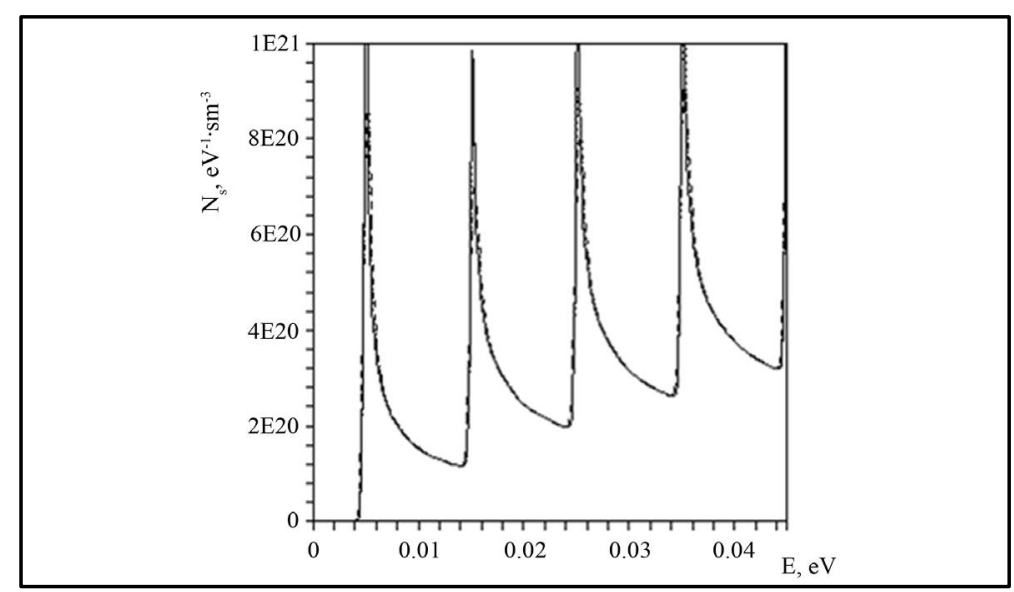

Figure 1. Density of energy states calculated using a series expansion in functions $G N$, function derivative Fermi-Dirac and Gaussian function in a strong magnetic field $H=10 \mathrm{kOe}$ at $T=0.4 \mathrm{~K}$.

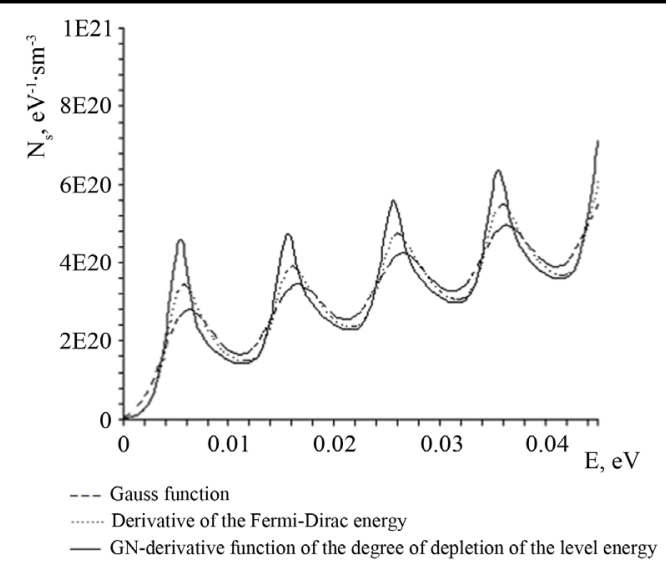

Figure 2. The temperature dependence of the density of states in a strong magnetic field ( $H=10 \mathrm{kOe})$ at temperatura $T=10 \mathrm{~K}$.

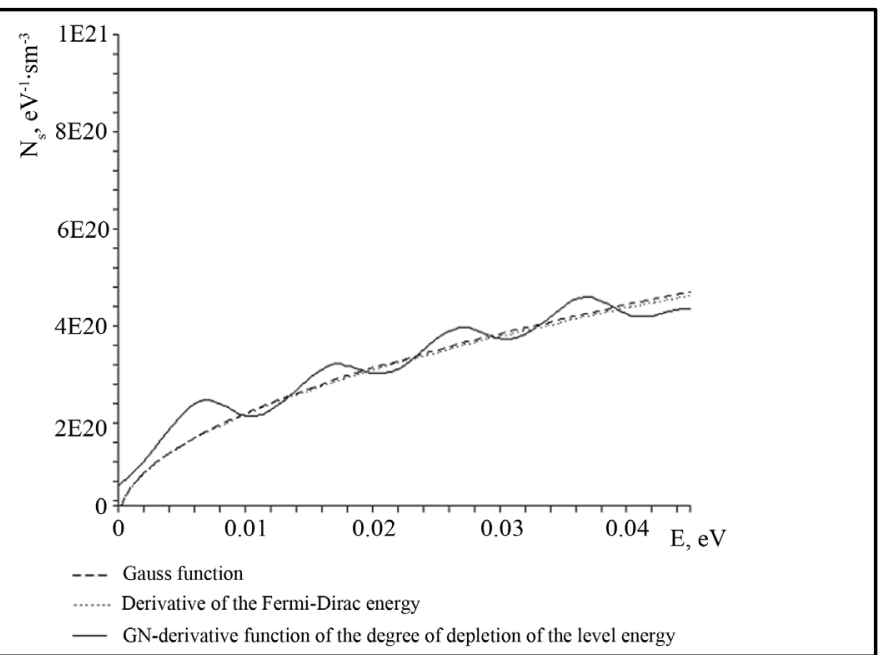

Figure 3. The temperature dependence of the density of states in a strong magnetic field $(H=10 \mathrm{kOe})$ at $T=35 \mathrm{~K}$. 
the same, then finding the density of states can be considered complete. Thus, measurements $N_{s}(E, H)$ for various values of $T$ and $H$ should complement each other and this should ensure the accuracy of the results.

\section{Analysis of the Experimental Results}

Analyze the density of the energy states of specific semiconductors in a quantizing magnetic field. Figure 4(a) shows the density of states $\mathrm{PbS}$ at temperature $T=77 \mathrm{~K}$ and a magnetic field $H=32 \mathrm{kOe}$ [11]. Within experimental accuracy, the density of states is continuous, monotonically increasing, smooth function of energy. In this figure, the oscillations of the density of states are observed. This indicates that $\hbar \omega_{c}<k T$. We decompose a graph density of states in a series of GN-functions. When the expansion will take perfect $N_{s}\left(E_{0}, H\right)$. However, in contrast to the ideal we consider the dependence of the electron mass energy. Peak height $N_{s}(E, H, T)$, we assume an energy-dependent. Then choosing coefficients in the sum before $N_{s}\left(E_{0}, H\right)$ customize theoretical $N_{s}(E, H)$ to the experimental, and, fitting parameter and are the coefficients of the $N_{s}\left(E_{0}, H\right)$. Further, in Formula (5) will reduce the temperature. Starting with $T=20 \mathrm{~K}$ smooth curve begins to oscillate and stands Landau levels (Figure 4). Thus at temperatures $k T \ll \hbar \omega_{c}$, Landau levels are beginning to stand out as sharply distinct peaks $N_{s}(E, T)$. In a strong magnetic field, the energy states of the continuous spectrum is strongly deformed and transformed into oscillating line. With increasing temperature, the discrete levels are washed away, and the density of states is transformed into a continuous spectrum. In the experiment, the density of states depends on the energy, temperature, and the effective mass. Thus, with increasing energy separation between, the peaks vary.

At constant effective mass dependence of the density of states of the energy is $N_{s}(E) \sim \sqrt{E-E_{c}}$ [9] [10]. If with increasing electron energy increases the effective electron mass, decreases the distance between Landau levels, the density of states curve moves toward larger values of the density of states and moves upward along the axis of the density of states. On the contrary, if $m$ decreases with increasing energy density of states curve moves down along the axis of the energy density of states.

Thus, the magnetic field moves in a plane state density $N_{s}-E$ even at temperatures where Landau levels are not visible due to thermal broadening.

Comparison between theory and experiment with different values of the magnetic fields is shown in Figure 4. As seen from Figure 4 with increasing tension $N_{s}(E, H)$ compressed in a lower energy and rises axially $N_{s}$. This means that the distance between Landau levels in different energy gaps are different.

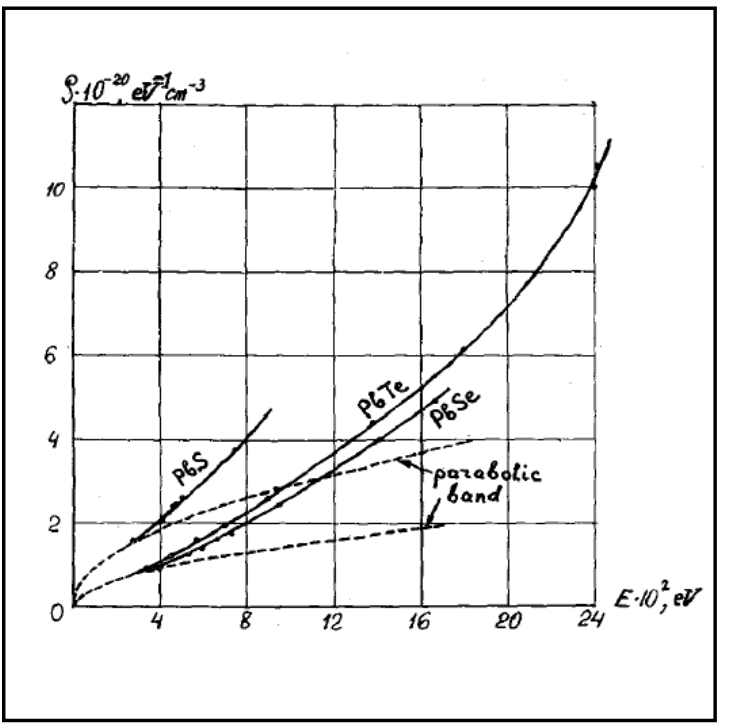

(a)

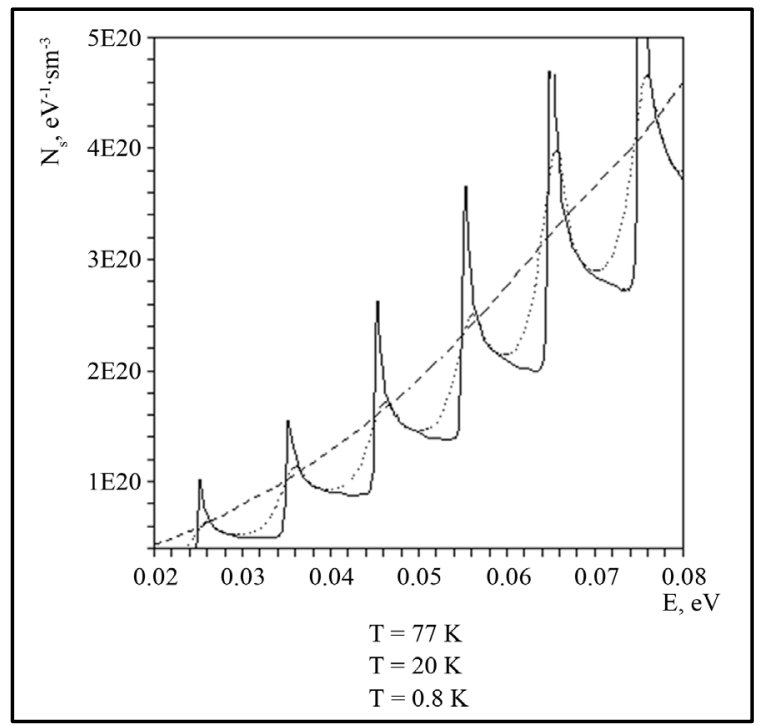

(b)

Figure 4. Changing the density of states with decreasing temperature in strong magnetic fields. (a) The density of states against the energy in the conduction band for an experiment in PbS, PbTe and PbSe at $H=32 \mathrm{kOe}, T=77 \mathrm{~K}$ [11]; (b) Model calculation of the density of states in a strong magnetic field for three different values of temperature for PbS. 


\section{Conclusion}

A model of the temperature dependence of the density of states in a strong magnetic field. The method of determination of the density of states in a quantizing magnetic field. Investigated the dependence of the density of states of the temperature. A parabolic dispersion law shows that the density of states in a strong magnetic field at a temperature increase coincides with the density of states in the sample without a magnetic field. It is shown that with increasing temperature, the Landau levels due to thermal broadening and washed away $N_{s}(E, H, T)$ converted into the density of states in the absence of a magnetic field. With the proposed model the experimental results $\mathrm{PbS}$ [11]. Using the proposed model of the high temperature, $N_{s}(E, H, T)$ calculated density of states at low temperatures. Simulation of the temperature dependence $N_{s}(E, H, T)$ possible to determine the Landau levels in $\mathrm{PbS}$.

\section{References}

[1] Tsidilkovsky, I.M. (1972) Electrons and Holes in Semiconductors. Nauka, Moscow.

[2] Bagraev, N.T., Brilinskaya, E.S., Gets, D.S., Klyachkin, L.E., Malyarenko, A.M. and Romanov, V.V. (2011) FTP (Fizika Technika Poluprovodnikov), 45, 1503-1508.

[3] Bagraev, N.T., Brilinskaya, E.S., Danilovsky, E.Yu., Klyachkin, L.E., Malyarenko, A.M. and Romanov, V.V. (2012) FTP (Fizika Technika Poluprovodnikov), 46, 90-95.

[4] Gulyamov, G., Sharibaev, N.Yu. and Erkaboev, U.I. (2013) Physical Surface Engineering, 11, 289-292.

[5] Gulyamov, G. and Sharibaev, N.Yu. (2011) FTP (Fizika Technika Poluprovodnikov), 45, 178-182.

[6] Gulyamov, G., Sharibaev, N.Yu. and Erkaboev, U.I. (2013) FIP (Fizicheckaya Injeneriya Poverkhnosti), 11, $195-198$.

[7] Gulyamov, G., Karimov, I.N., Sharibaev, N.Yu. and Erkaboev, U.I. (2010) Uzbek Journal of Physics, 12, $143-146$.

[8] Ziman, J. (1974) Principles of the Theory of Solids. Wiley, New York.

[9] Brandt, N.B. and Kulbachinsky, V.A. (2007) Quasiparticles in Condensed Matter Physics. Fizmatlit, Moscow.

[10] Lifshitz, I.M., Gredeskul, S.A. and Pastur, N.A. (1982) Introduction to the Theory of Disordered Systems. Nauka, Moscow.

[11] Ravich, Yu.I. (1968) Journal de Physique, 4, 114-124. 